Sociologie et sociétés

\title{
La United Kingdom Stem Cell Bank
}

Un petit espace stérile pour l'homme, un gigantesque espace multilatéral pour l'humanité

\section{The United Kingdom Stem Cell Bank}

\section{One Small Sterile Space for Man, One Giant Multilateral Space for Mankind}

\author{
Virginie Tournay, Marie-Odile Ott, Dörte Bemme et Christelle Routelous
}

Volume 42, numéro 2, automne 2010

Quand le vivant devient politique : les avatars de la démocratie technique

Making the Living a Policy Issue: The Embodiments of Technological Democracy

URI : https://id.erudit.org/iderudit/045365ar

DOI : https://doi.org/10.7202/045365ar

Aller au sommaire du numéro

Éditeur(s)

Les Presses de l'Université de Montréal

ISSN

0038-030X (imprimé)

1492-1375 (numérique)

Découvrir la revue

Citer cet article

Tournay, V., Ott, M.-O., Bemme, D. \& Routelous, C. (2010). La United Kingdom Stem Cell Bank: un petit espace stérile pour l'homme, un gigantesque espace multilatéral pour l'humanité. Sociologie et sociétés, 42(2), 291-312.

https://doi.org/10.7202/045365ar

\section{Résumé de l'article}

L'objectif de cette contribution est d'étudier le lien entre l'organisation matérielle confinée de recherches émergentes autour du vivant, leur consolidation disciplinaire et le déploiement d'espérances collectives à grande échelle. Le cas d'étude est la United Kingdom Stem Cell Bank, décrite comme une architecture performative dotée d'une efficacité organisationnelle et jouant un rôle clé dans la gouvernance internationale de la manipulation des cellules souches embryonnaires humaines. L'enjeu consiste à montrer que la structuration de cet espace confiné et stérile, nécessaire au maintien des cellules souches embryonnaires humaines, est également celle d'un espace de coopérations multilatérales et internationalisées autour de ces produits biologiques. Pour étudier cette tension scalaire, la différenciation institutionnelle de la UK Stem Cell Bank est appréhendée comme une catégorie de mouvement d'individus, de produits biologiques et de données les concernant. En intégrant les perspectives récemment développées autour des policy transfer studies, cette contribution met l'accent sur le mouvement régulier, répété et centralisé de collecte de cellules d'origine humaine vers cet espace confiné, ainsi que sur les procédures d'étalonnage et d'enregistrement des données pour expliquer le succès de ce modèle local à grande échelle.
Tous droits réservés @ Les Presses de l’Université de Montréal, 2010
Ce document est protégé par la loi sur le droit d'auteur. L’utilisation des services d’Érudit (y compris la reproduction) est assujettie à sa politique d'utilisation que vous pouvez consulter en ligne.

https://apropos.erudit.org/fr/usagers/politique-dutilisation/ 


\title{
La United Kingdom Stem Cell Bank
}

\author{
Un petit espace stérile pour l'homme, \\ un gigantesque espace multilatéral pour l'humanité
}

\section{VIRGINIE TOURNAY \\ CNRS \\ Institut d'études politiques de Grenoble \\ UMR PACTE \\ MEOS (Université de Montréal) \\ BP 48, 38040 Grenoble Cédex 9, France \\ Courriel: virginie.tournay@iep-grenoble.fr}

\section{MARIE-ODILE OTT}

\section{INSERM}

EDQM Health care activites

European Directorate for the Quality of Medicines

\& HealthCare

Council of Europe; 7, allée Kastner,CS 30026 ,

F-67081 Strasbourg, France

Courriel: marie-odile.ott@edqm.eu

\section{DÖRTE BEM ME}

Faculté des sciences infirmières

et MEOS

Université de Montréal

C.P. 6128 , succursale Centre-ville

Montréal (Qc) $\mathrm{H}_{3} \mathrm{C} 3 / 7$

Courriel: doerte.bemme@umontral.ca

\section{CHRISTELLE ROUTELOUS}

Institut du management

École des hautes études en santé publique

Av du Pr. Léon Bernard CS 74312

35043 Rennes Cédex, France

Courriel: christelle.routelous@ehesp.fr

$\ll \bigcup \begin{aligned} & \text { N PETIT PAS POUR L'HOMME, UN GRAND PAS POUR L'HUMANITÉ». C'est avec cette } \\ & \text { petite phrase vouée à l'histoire que l'astronaute Neil Armstrong pose le pre- }\end{aligned}$ mier pied terrestre sur le sol lunaire le 20 juillet 1969. Symbolisant les gigantesques retombées scientifiques et les progrès technologiques que la conquête de l'astre lunaire laisse supposer par la foulée humaine d'une de ses excavations, cette formulation brève ne semble avoir qu'un rapport relativement éloigné avec l'objet de notre propos qui concerne une autre forme de conquête: celle de l'infiniment petit biologique liée à la maîtrise des produits dérivés des embryons humains. Dans ce cas de figure, le périple collectif n'est pas spatial mais médical; il vise à maîtriser ce qui figure comme les composants biologiques les plus chargés symboliquement. Expressions immédiates de la vie dans ses balbutiements, l'embryon humain et ses produits dérivés sont les 
ingrédients de prédilection pour ancrer irrémédiablement le rêve pluricentenaire d'une médecine qui permettrait de réparer, de remplacer pièce par pièce, les éléments défectueux ou usés de la machine humaine. La fondation de cette médecine réparatrice remonte aux premières greffes d'organes et elle se prolonge par les techniques d'ingénierie tissulaire dont l'objectif est de façonner par l'intermédiaire de cultures in vitro, certains éléments organiques faisant l'objet de greffes, tels que la peau, les vaisseaux, l'os ou la cornée. La caractérisation et la disponibilité des cellules embryonnaires ou fotales définissent ainsi un enjeu puissant: la consolidation des thérapies cellulaires à des fins régénératrices.

Si l'on porte attention aux équipements qui prennent forme ces dernières années autour des produits composés de cellules embryonnaires, le message proverbial formulé par Neil Armstrong à son arrivée sur le sol lunaire trouve peut-être une parenté dans ce qui augure une médecine régénératrice à base de cellules embryonnaires humaines ${ }^{1}$. Il est vrai que l'isolement des premières lignées ${ }^{2}$ embryonnaires humaines n’a pas été suivi en direct par un milliard de personnes rivées derrière leur écran de télévision comme cela a été le cas pour la conquête lunaire. Pourtant, avec les espoirs suscités par cette potentielle médecine régénératrice, de nouvelles formes de solidarités sociales se sont organisées autour des personnes qui présentent de lourdes pathologies. Ces alliances s'appuient sur les usagers de soins candidats à des traitements basés sur des cellules souches humaines pour répondre à des maladies neurodégénératives $d u$ type Alzheimer, Parkinson, des scléroses en plaques ou des accidents irréversibles touchant la colonne vertébrale. Des associations de patients se mobilisent et des nouvelles cliniques spécialisées surgissent dans différents pays, des fonds sont distribués et des programmes sont proposés par les pouvoirs publics à des échelles régionales, nationales, communautaires ou internationales. Véhiculant de fortes recompositions sociales, ces solidarités biologiques correspondent à une véritable «biosocialité» pour reprendre le terme de Paul Rabinow (1994). Ces nouvelles trajectoires cellulaires inscrivent ainsi de nouveaux modèles d'échelle biologique, de temporalité des soins et de connexions bioculturelles au corps (Franklin, 2005).

Mais que le lecteur ne s'y trompe pas. Notre propos ici n'est pas de discuter du bien-fondé des espérances suscitées par cette orientation thérapeutique, mais plutôt de comprendre en quoi l'organisation matérielle des recherches autour des embryons humains véhicule avec elle toute une cohorte d'enjeux multi-scalaires, allant de la simple concurrence inter-équipe dans l'établissement des premières lignées embryonnaires stabilisées, à la formulation de controverses sociétales largement médiatisées ou à la mise en place de programmes internationaux nécessitant des financements consé-

1. Cela n'exclut pas d'autres perspectives concurrentes en cours de développement telles que les induced pluripotent stem cells qui consistent à produire des modèles thérapeutiques à partir de cellules adultes directement prélevées chez le patient.

2. Une lignée cellulaire est une population homogène stabilisée de cellules issues des divisions successives. Elle détient théoriquement une capacité illimitée de division. 
quents ainsi que des partenariats multilatéraux consolidés. Si l'hypothèse d'une coconstruction des objets techniques et de leurs politiques d'encadrement constitue aujourd'hui un postulat fort de la sociologie des controverses scientifiques et plus largement des science studies, les interrelations entre la constitution d'agencements matériels et le façonnement des subjectivités individuelles demeurent quant à elles, faiblement étudiées (Tournay et Leibing, 2010). Cette lacune provient de la tendance à définir les manifestations d'espoir et d'engouement à l'égard d'une conquête technologique, comme des propriétés liées à des subjectivités individuelles isolées ou, au contraire, à les rattacher à des propriétés objectives et aux performances des produits technologiques quand l'analyse sociologique s'adresse à de larges collectifs et porte sur l'acceptabilité sociale de technologies grandement médiatisées. L'hypothèse développée dans cet article est de considérer que l'ancrage disciplinaire internationalisé des recherches autour des cellules souches embryonnaires humaines [CSEh] et leur rationalisation progressive reposent sur la structuration de la UK Stem Cell Bank [UKSCB] qui débute en $2003^{3}$. Ces activités de banking autour des produits cellulaires (que l'on peut traduire littéralement par «mise en banque») relèvent des régulations mises en place dans les sciences biomédicales (bases de données) pour contrôler la circulation de divers matériels biologiques (tels que les divers composants du sang, les protéines, les supports matériels de la cartographie et du séquençage génomique) dans le cadre du Human Genome Project des années quatre-vingt-dix (Hilgartner, 1995; Peerbaye, 2004; Gaudillière et Rheinberger (dir.), 2004). Dans la lignée de ce que nous allons développer, Stephens, Atkinson et Glasner (2008) montrent que l'organisation de la UKSCB s'appuie sur une architecture performative qui assoit la légitimité des pratiques de stockage et de circulation du matériel biologique à base de cellules souches au sein de la communauté scientifique internationale. Pourtant, un bref regard sur cette structure montre que son efficacité organisationnelle est loin d'être proportionnelle à la disponibilité matérielle et humaine de cette installation. En regroupant moins d'une vingtaine de personnes employées dans l'équivalent d'un petit immeuble de la banlieue londonienne, la UKSCB forme aujourd'hui un agencement coopérateur responsable de l'institutionnalisation disciplinaire de ce qui jusqu'alors n'était qu'un ensemble de recherches éparpillé en Grande-Bretagne et au-delà. Le rôle central de la banque dans l'économie de la circulation des cellules souches embryonnaires humaines contraste ainsi fortement avec les faibles moyens initialement engagés, la petitesse et le caractère provisoire des locaux prévus à cet effet. En 2008, la banque ne comprend

3. Les raisons pour lesquelles cet espace de coopérations internationale commence en GrandeBretagne mériteraient un travail spécifique. Plusieurs pistes sont à explorer. La permissivité juridique à l'égard de ces produits biologiques, la grande attention accordée à la dimension de sécurité sanitaire des produits, de même que les traditions locales de pratiques bureaucratiques sont sans doute des ingrédients qui ont exercé une certaine attractivité sur les équipes de recherche du monde entier. En outre, des arguments organisationnels interviennent: le caractère public de la banque sans but lucratif, ainsi que le dépôt obligatoire des lignées cellulaires nationales contribuent indéniablement à une attractivité. 
pas plus d'une quinzaine de personnes, incluant la secrétaire ${ }^{4}$. Loin de développer spécifiquement une politique de relations avec l'extérieur, l'essentiel des membres de la banque se trouve plutôt impliqué dans le contrôle de la qualité ou au niveau des aspects techniques liés au développement des méthodes de culture. L'objectif est d'établir un équipement et des procédures robustes permettant de stabiliser les lignées de cellules embryonnaires en culture, de conserver leurs propriétés biologiques et d'assurer un usage clinique sans danger (Healy et al., 2005).

On observe ici un contraste saisissant entre le confinement de l'espace, le peu de ressources consacrées à la préservation du matériel biologique d'une part, et la très forte légitimité symbolique ainsi que les procédures contraignantes de gouvernance généralisées à l'ensemble de la communauté internationale découlant de son fonctionnement d'autre part. Cette tension scalaire entre un univers matériel confiné, mobilisant un faible nombre d'acteurs locaux, et un large public, constitué par l'ensemble des individus et des équipes destinataires de ces pratiques confinées, se retrouve également dans le programme spatial Apollon 11. Qu'il s'agisse pour l'homme d'effleurer le sol lunaire ou de rendre immortelles des cellules embryonnaires, c'est avant tout la démonstration de la maîtrise d'un environnement auparavant considéré comme indomptable ou inaccessible. La sortie du module lunaire des astronautes de la mission Apollon 11 se résume à l'intervention d'un équipement minimal: ordinateurs de bord, pilotage automatique, combinaisons spatiales, caméras d'enregistrement, postes terrestres de réception des informations de la NASA, ainsi que d'une vingtaine de personnes localement investies dans le recueil immédiat d'informations. En dépit de ce confinement, l'évènement fut suivi par un public estimé à un milliard de téléspectateurs. Ainsi, ces deux formes de conquêtes disciplinaires: spatiale et médicale, peuvent être rapprochées au niveau de la tension scalaire particulière à leur agencement. On peut également mettre en lien ces démonstrations de maîtrise technologique à partir des formes d'espérance qu'elles colportent. Ainsi, les assemblages matériels déployés à partir de ces deux formes de conquête n'incarnent pas des montages technologiques qui détiendraient intrinsèquement une efficacité particulière à grande échelle ou une certitude d'avancée sociétale ontologiquement fondée. La capsule Apollon 11 et la UKSCB renvoient à ce que l'un des contributeurs de cet article définit comme des architectures de monstration (Tournay, 2009a), c'est-à-dire des agencements qui «attachent», qui démontrent par leur structure même, la visée d'espoir laquelle demeure néanmoins constitutivement incertaine dans son accomplissement. Quelles que soient leurs tailles, ces assemblages matériels innovateurs nécessitent un haut degré d'espérance, et les scénarisations du futur s'avèrent indissociables des pratiques concrètes développées au quotidien. Ces attentes collectivement partagées ne reposent pas sur l'accessibilité de la technologie. Pire encore, plus les individus sont territorialement éloignés de la source de production des connaissances et des dispositifs tech-

4. Interviewé par nos soins en février 2008, le directeur de la banque Glyn Stacey ne souhaite alors pas étendre le recrutement à l'exception d'un poste de business development, les fonds nécessaires n'étant pas disponibles. 
niques, plus les promesses qui entourent les recherches sur les cellules souches embryonnaires ressortent de manière puissante, assurée et univoque (Brown, 2003: 16). Aussi, la difficulté à penser les rapports entre la fabrique d'un assemblage matériel et la construction collective de l'attente n'est pas simplement reliée à une différence de nature entre ce qui relèverait de la matérialité et ce qui serait de l'ordre de la subjectivité. Elle s'avère inextricable d'une tension scalaire, c'est-à-dire de l'écart des focales d'observation entre les bricolages technologiques contenus dans le laboratoire, ou plus généralement dans toute enceinte confinée, et la constitution ouverte de leurs controverses et débats. Le périmètre étendu des publics concernés tranche ici considérablement avec l'espace concentré de la banque britannique et les localisations territoriales contingentées des produits de cellules embryonnaires humaines.

L'objectif de cette contribution est de montrer comment la structuration d'un espace confiné et stérile, nécessaire au maintien des cellules souches embryonnaires, a également été celle d'un espace de coopérations multilatérales et internationalisées autour de l'économie de ces produits biologiques. Comprendre ces tensions scalaires nécessite de modifier radicalement notre regard en appréhendant les espaces et les emplacements qui ne sont pas des concepts statiques invariablement délimités par des frontières sur lesquelles tout le monde s'accorde (Eriksson, Stephens, Webster, 2008). L'idée est de définir l'espace artificiellement construit comme une catégorie de mouvements individuels, de produits biologiques et de données les concernant (Tournay, 2008 et 2009b). L'espace peut ainsi être simultanément appréhendé à titre de culture matérielle particulière, d'un point de passage à la circulation de dispositifs matériels et narratifs et/ou d'un mode singulier de production de solidarités collectives appuyé sur une instrumentation spécifique (Lascoumes et Le Galès, 2005). Le plan adopté dans cet article vise à caractériser ce mouvement spécifique rendant l'ancrage territorial bien circonscrit de la UKSCB internationalement performant, c'est-à-dire concourant à la consolidation disciplinaire et à la constitution d'une espérance collective généralisée autour des cellules souches humaines ${ }^{5}$. Dans cette optique, il nous faut d'abord dresser un inventaire des acteurs, des dispositifs techniques et des procédures impliqués dans la différenciation institutionnelle de la UKSCB avant de nous intéresser plus spécifiquement à la circulation internationalisée des lignées cellulaires, aux alignements à grande échelle des pratiques liées à ces produits ainsi qu'aux processus de traçabilité et de conservation des informations. En mettant l'accent sur les arrangements matériels observés depuis une échelle microsociologique, la conclusion est l'occasion de discuter la manière dont une institution initialement nationale devient progressivement un agencement internationalisé, susceptible de consolider le champ disciplinaire émergent des recherches autour des cellules souches embryonnaires humaines [CSEh].

5. Cette étude de cas repose sur une série d'entretiens semi-directifs effectués auprès des membres de la UKSCB en février 2008 par le premier auteur de cet article ainsi que sur des documents internes fournis par ces mêmes acteurs, concernant le fonctionnement et la régulation de cette banque. Elle a bénéficié d'une mise en perspective et d'un enrichissement par les discussions conduites avec les co-auteurs engagés dans des projets complémentaires relatifs aux traitements politiques des cellules souches. 
Suivre les conditions entourant le stockage et l'accès aux données peut ainsi contribuer à éclairer la question de la coopération scientifique internationale, et par là même les analyses de politique publique (Hilgartner et Brandt-Rauf, 1994) marquées par cette échelle d'observation. L'enjeu heuristique consiste à saisir la façon dont les principes de régulation des produits mis en œuvre dans cette banque ont pu s'imposer dans des contextes culturels et historiques variés. Ce cas d'étude concerne donc la question du transfert politique, c'est-à-dire la circulation de modèles dans des contextes hétérogènes. L'article de Dumoulin et Saurugger (2011) montrant l'intérêt de combiner les approches centrées sur les instruments de l'action publique et la sociologie de la traduction pour rendre compte des mécanismes de transfert sert ici de point d'appui à la discussion de cette dynamique institutionnelle. En effet, la croisée de ces deux approches suggère que la UKSCB ne peut pas être envisagée comme une organisation performante en soi, dont les caractéristiques ont été posées a priori de sa mise en œuvre, mais plutôt comme l'aboutissant momentané d'un processus d'enrôlement et de persuasion, inscrivant dans ce mouvement même des intérêts et une efficacité partagés pour la communauté internationale travaillant sur les CSEh. L'ouverture aux approches des politiques publiques par les instruments et la théorie de l'acteur-réseau (actor network theory), préconisée par les deux auteurs, permet de rendre compte de la circulation de modèles institutionnels et de la généralisation des normes, en intégrant l'idée d'une coévolution des pratiques, des cadres de référence et des projets. Cela présuppose l'absence d'orientations préconçues des processus de différenciation et d'intégration institutionnelles (Saurugger, 2010) ${ }^{6}$. En d'autres termes, ce présent article est un plaidoyer visant à porter une attention plus soutenue au déploiement concret des activités institutionnelles dans leurs ramifications les plus lointaines (White, 2010 $)^{7}$, sans supposer de frontières aux collectifs que l'on définit ou de projets préconçus aux acteurs que l'on étudie (Schemeil, 2009).

\section{LE RÔLE CENTRAL DE LA UKSCB DANS LA GOUVERNANCE INTERNATIONALE AUTOUR DES CSEH}

La régulation de la production des lignées de CSEh s'appuie sur un cadre juridique complexe $^{8}$ car elle conjugue l'application de contraintes réglementaires nationales et des bonnes pratiques professionnelles relativement aux produits d'origine humaine et à la culture des cellules souches. Elle prend en compte les filières de transformation du

6. Pour un état de la production théorique autour des études européennes et une discussion approfondie sur les manières d'appréhender les organisations internationales.

7. Ce constat va dans le même sens que de récents travaux centrés sur l'appréhension de l'européanisation. Ainsi, pour White, comprendre l'intégration européenne implique de ne pas contraindre le regard aux seuls représentants du parlement européen mais suppose également de suivre les activités et les débats au-delà des institutions bruxelloises, véhiculés par tous citoyens.

8. Elle fait intervenir à un niveau national: Human Fertilisation and Embryology Act (1990), Human Fertilisation and Embryology Research Purposes Regulations (2001), Human Tissues Act (2004), Human Tissues Regulations (Quality et Safety for Human Applications) (2007); et au niveau communautaire Tissues and Cells Directive (2004/23/EC et 2006/86 et 2006/17). 
produit où interviennent l'origine de la souche (embryonnaire, fotale ou adulte), le type d'utilisation concrète (laboratoire de recherche, perspective clinique, produit manufacturé) ainsi que l'intention officielle, c'est-à-dire la programmatique de recherche. Il faut distinguer les travaux de recherche autorisés des stockages pour un usage futur indéterminé. En février 2008, la UKSCB dispose de plus d'une soixantaine de lignées de CSEh approuvées, provenant de Grande-Bretagne et d'ailleurs, chacune située à différents niveaux du processus de banking et du contrôle qualité.

Un bref regard sur les activités de la banque montre que ces démarcations juridiques, liées à la diversité des usages attendus de ces produits, traduisent plutôt un certain continuum de pratiques. Bien qu'on observe une grande variabilité des méthodes d'obtention mises en œuvre par les différentes équipes de recherche qui déposent des lignées de cellules embryonnaires, l'ensemble de la collection est soumis au même processus d'assurance et de contrôle de la qualité. Ainsi, le mouvement général de circulation des lignées cellulaires (c'est-à-dire l'organisation des étapes d'entrée, de préparation et de la chaîne de production) n'est pas considérablement affecté par la destination supposée de ces produits. Il nécessite néanmoins des organismes intermédiaires spécifiques pour de la délivrance des autorisations.

Pour les promoteurs de la banque, l'objectif est de constituer une collection représentative des différents centres nationaux et internationaux travaillant sur ces produits biologiques d'une part, et des différents types de modifications réalisées sur les cellules souches embryonnaires d'autre part (les chercheurs parlent d'une nouvelle dérivation pour qualifier l'établissement d'une lignée de cellules embryonnaires génétiquement distincte d'une autre). Le cadre réglementaire déborde ainsi des contours institutionnels stricts de la UKSCB puisque la fonction de la banque est d'accepter, de conserver et de délivrer des produits insérés dans une chaîne de production dont les points de contrôle figurent dans un espace d'abord et avant tout national. La différenciation institutionnelle de la UKSCB inclut donc de nombreux acteurs ainsi que des dispositifs techniques, initialement extérieurs à son émergence, mais qui, progressivement, en composent sa réalité et en déterminent sa gouvernance. Cette convergence administrative s'est constituée dès l'amorçage de la banque par la nécessité d'apposer des normes professionnelles à des produits partagés par différents établissements. Ce mécanisme de différenciation passe avant tout par un processus volontaire plutôt que contraignant, il est le résultat d'un étalonnage administratif interinstitutionnel plutôt que le résultat d'une politique administrative cohérente (Olsen, 2003). Ainsi, la banque reçoit du matériel issu des évaluations médicales réglementées de donneurs potentiels, elle mobilise le concours de laboratoires fournisseurs de lignées dérivées, accrédités pour leurs équipements et leurs systèmes de gestion de la qualité. Elle intègre des activités de stockage à des fins de recherche ou à destination d'éventuelles perspectives de contrôle clinique, de caractérisation et de gestion sanitaire des lignées, suivant des conditions et des équipements accrédités GMP, assurant une traçabilité des lignées stockées ainsi que la mise en œuvre d'un système d'alerte. Cet espace miniature de la UKSCB est extrêmement ramifié, il reçoit des échantillons biologiques provenant de destinateurs multiples et il envoie des informations vers tout autant de destinataires. 
Le personnel de la UKSCB vise à constituer un réservoir de qualité clinique adapté à un usage thérapeutique. Cet environnement de grade clinique ne diffère pas de celui des banques de grade recherche si on compare les procédures ou l'organisation des étapes de stockage, de préparation et de croissance du matériel biologique. Les promoteurs de la banque projettent une installation plus grande et permanente afin de stocker et de distribuer les premières lignées cellulaires ayant satisfait au contrôle qualité; ils s'inscrivent dans le plus long terme et mettent en place des lignées cellulaires de grade clinique. L'installation d'une filière de production à grade clinique constitue donc un enjeu actuel fort. En dépit du fait que la banque soit loin de pouvoir mettre en place des produits directement injectables aux patients, la contiguïté organisationnelle, entre la mise en banque à orientation recherche et à orientation clinique naturalise la structuration d'espérances collectives vers de possibles applications médicales. Tout comme la bio-économie de promesses formée autour des banques de sang de cordons (Martin et al., 2008), la UKSCB matérialise un régime de l'espoir à travers la coconstruction d'un ensemble de projections thérapeutiques et d'une chaîne de préparation des produits cellulaires dont les modalités techniques demeurent encore fortement débattues (Hunt, 2007).

\section{Un espace restreint pour des produits à finalité multiple et une gouvernance dispersée}

Différents organismes ponctuent la collecte des lignées cellulaires en y apposant des conditions. La Human Fertilisation and Embryology Authority (HFEA) est la plus importante. Cet organisme délivre des licences aux groupes de recherche britanniques qui génèrent des lignées de cellules souches embryonnaires. L'obtention de cette licence de dérivation est conditionnée par le fait que les lignées embryonnaires constituées en Grande-Bretagne doivent obligatoirement être déposées à la UKSCB. La HFEA couvre les recherches, les utilisations d'embryons ainsi que la dérivation des CSEh. La préparation de ces produits biologiques est régulée par cet organisme jusqu'au stade de l'établissement de la lignée cellulaire déposée à la UKSCB.

Lorsque la lignée cellulaire est admise à la banque, elle passe ensuite sous l'autorité du comité de pilotage (Steering Committtee — SC), deuxième point de passage obligé à ce mouvement d'intégration du produit biologique. Le SC est une organisation non gouvernementale qui assure la supervision éthique des lignées dérivées stockées à la UKSCB suivant le cadre réglementaire de leur utilisation et de leur circulation. Ainsi, l'envoi et le transfert des lignées cellulaires doivent être approuvés par le SC. Il supervise les lignées souches à orientation de recherche et il observe les utilisations de lignées cellulaires à usage clinique.

La Human Tissue Authority (HTA) est instaurée en 2004. Cette institution est le point de passage obligé pour les activités de mise en banque. Elle régule le prélèvement, le stockage et l'utilisation des cellules humaines, notamment des composants sanguins et de la moelle osseuse. Ce domaine de compétences est élargi en 2007 par l'inclusion des lignées de cellules souches et somatiques. On observe ainsi un léger recoupement 
des domaines de compétences de la HFEA et de la HTA concernant les lignées embryonnaires nouvellement dérivées. Elle délivre également la licence pour les équipements britanniques relatifs aux prélèvements, à la transformation, au stockage et à la distribution des cellules humaines destinées à des applications relevant de la directive européenne Tissus et cellules. En d'autres termes, cette organisation recouvre la totalité du matériel relatif aux applications cliniques n'étant pas sous la régulation de la HFEA. Elle inspecte tous les établissements travaillant avec des tissus humains. Elle joue donc un rôle particulièrement important dans la régulation des activités de mise en banque. Sous couvert de cette autorisation, le personnel de la UKSCB tente d'automatiser des cultures cellulaires au niveau de la distribution des produits cellulaires. Cela justifie l'utilisation d'un bioréacteur, qui est un équipement lourd, ainsi que des collaborations fortes au sein de l'Union européenne. Les effets concrets de l'application des directives en cours sont encore loin d'être tous prévisibles (Hoeyer, 2010).

La Medicines and Healthcare-products Regulatory Agency (MHRA) est le point de passage obligé des cellules destinées à devenir un produit manufacturé, c'est-à-dire de celles devant s'intégrer aux équipements de fabrication. Pour obtenir son aval, la UKSCB doit envoyer le matériel humain aux organismes de régulation et fournir une analyse certifiée du matériel (nature, contrôles effectués, destination) à partir de laquelle la licence est établie. La banque a ainsi reçu une accréditation par la MHRA pour son installation aux conditions GMP ainsi que des licences pour ses équipements.

La délivrance d'autorisations pour la mise en culture et le stockage des lignées cellulaires forme une première barrière à l'entrée de produits homologués et caractérisés par le personnel de la UKSCB. Le personnel de la banque a développé un deuxième ensemble d'épreuves appuyé sur un système de gestion de la qualité ainsi que sur la standardisation de matériels de référence pour la culture des lignées de cellules souches et de ses dérivés. Une activité d'expertise a ainsi pris forme auprès de différents acteurs institutionnels et privés, ce qui contribue au rayonnement national et international de la banque. De nombreux organismes de régulation investis dans la fabrication de produits à base de cellules humaines sollicitent la banque afin de paramétrer leur régime de contrôle et éventuellement leur processus de distribution des produits manufacturés (cela mobilise plus de quatre-vingts organismes différents dans le monde, dont certains organismes internationaux tels que la MEA ou le WHO). Le personnel de la banque évalue différents milieux pour la croissance des lignées cellulaires à destination des compagnies privées ainsi que pour l'application de supports plastiques à de multiples lignées de cellules humaines afin de suivre leur prolifération. L'objectif est de produire une méthodologie générique au développement des CSEh, à partir de différents milieux de culture. En plus de ces organismes, le personnel de la banque renseigne également différentes équipes au sujet de leurs résultats de recherche, notamment sur les questions de confidentialité et sur l'absence d'implication de la banque britannique dans les intérêts commerciaux ou intellectuels. Il fournit du matériel de référence et il est consulté pour l'extension de contrôles existants à de nouveaux produits ou à la prise en charge d'évènements indésirables liés à un produit cellulaire. 
La banque ne retire cependant aucun bénéfice financier, elle ne retient pas de propriété intellectuelle sur la coopération menée avec les compagnies privées. Dans le cas de découvertes susceptibles d'avoir des retombées économiques considérables, par exemple l'obtention d'une méthode efficace de culture, elle est contrainte de discuter avec le Medical Research Council et les bénéfices de la découverte (les redevances) sont versés à ce même organisme, qui devra utiliser cet argent pour assurer le bon fonctionnement de la banque. Bien qu'elle ne soit pas posée explicitement, cette organisation institutionnelle rejoint la constellation imaginaire du service public (Hastings, 1999). Elle constitue une manifestation de la puissance publique ${ }^{9}$ (Hauriou, 1892) puisque le cadre d'actions de la UKSCB consiste à faire exister un collectif: celui des chercheurs travaillant sur les CSEh, en créant le besoin d'un service: la délivrance de produits embryonnaires conformes aux propriétés univoques et bien caractérisées.

\section{Une traçabilité confidentielle des informations pour un espace internationalisé}

L'intervention internationale de la UKSCB ne se limite pas à l'établissement de collaborations autour de grands projets d'équipements, ni même aux fonctions de conseiller que nous venons de mentionner. La banque participe activement à un travail d'étalonnage à grande échelle des méthodes de culture adaptées aux lignées cellulaires. Cette opération est menée dans le cadre de l'International Stem Cell Initiative Project dont l'objectif est de tester et de maintenir la stabilité génétique des cellules humaines à partir de différents milieux de culture.

Le processus de stockage et l'organisation de la culture au sein de la banque britannique répondent aux exigences méthodologiques du déposant, ce qui a pour effet une variabilité des conditions de culture elle-mêmes dépendante des critères de chaque groupe portant leur lignée. Pour favoriser un alignement de ces conditions, l'expérimentation internationale s'appuie sur une dizaine de milieux contrôlés qui sont en cours d'évaluation. L'opération est délicate car les CSEh ont des caractéristiques qui varient fortement dans le temps et suivant le nombre de générations. Seuls sont retenus les milieux de culture qui répondent aux meilleures conditions de croissance des cellules. À cette fin, le personnel de la UKSCB s'appuie sur des lignées cellulaires nouvellement conservées au sein de la banque (qui forment la banque "pré-master») et testées sur des milieux de culture sélectionnés. L'objectif est de mettre en place une assurance générale de la qualité des milieux cellulaires (Stacey, 2004), et, au-delà, de définir une nouvelle banque de distribution standardisée au niveau du contrôle des milieux de culture. Une quantité impressionnante de données relatives aux lignées est stockée et de nombreux échantillons sont produits en vue d'une distribution aux chercheurs. Cette méthodologie est automatiquement réalisée à partir des échantillons de la banque «pré-master» afin de préserver et de fournir un matériel de qualité égale.

La UKSCB participe activement à l'International Stem Cell Banking Initiative [ISCI] (ISCI, 2009) dont l'objectif est de coordonner les banques nationales qui dis-

9. Il apparaît ici que le service public est une composante de la puissance publique (Hauriou, 1892). 
tribuent les lignées de cellules souches humaines. À terme, il s'agit d'émettre un ensemble de guides de bonnes pratiques permettant à la banque de standardiser la distribution de ce type de matériel. L'ISCI illustre la relation étroite qui existe entre l'établissement d'un réseau collaboratif et la stabilisation d'un champ disciplinaire émergent autour des CSEh, en exigeant l'acquisition d'un équipement standardisé, pour limiter les incertitudes liées à la multiplicité des chaînes de production biologique (Webster et Eriksson, 2008). Il est en effet impossible de standardiser la croissance, les propriétés et les usages des produits cellulaires dans leur diversité. La coordination prend place autour d'un cadre partagé et contrôlé de procédures de caractérisation, de mise en culture et de préservation des lignées. On peut dire que la UKSCB assure véritablement la gestion de l'incertitude biologique dans un espace contrôlé (Rheinberger, 1997); elle s'apparente en d'autres termes à un modèle institutionnel à essai, éventuellement reproductible dans d'autres arènes.

L'espace internationalisé de la UKSCB se constitue également à partir du système de gestion de la traçabilité et de la sécurité. Suivant la directive européenne 2004/23/ EC, 2006/86 et 2006/17 qui régule la sécurité et la qualité en matière de Tissus et cellules (d'origine humaine), la banque doit tracer totalement le produit recueilli depuis le donneur jusqu'à son destinataire. L'évaluation médicale du donneur doit être recueillie et un contrôle microbiologique et de la qualité du produit biologique doit être effectué. La procédure de traçabilité liée aux conditions de culture, en particulier l'archivage d'informations - est particulièrement importante dans le cas d'effets indésirables relatifs aux essais cliniques et surtout aux greffes. Cette traçabilité passe par un système spécifique d'identifications et de correspondances. Dans le cas «traditionnel» des banques de tissus britanniques, le dossier donneur comporte les informations biologiques permettant d'établir le lien avec le produit biologique engagé dans le processus de préparation (numéro d'identification). Des dispositions d'identification assurent la traçabilité et les évaluations de risque à différentes étapes de préparation du produit biologique, depuis le donneur jusqu'au receveur. En d'autres termes, pour les banques de tissus et de cellules destinées à un usage thérapeutique, un seul identifiant accolé au produit suffit à autoriser le stockage et la préparation du produit, que celui-ci soit destiné à être réinjecté au donneur ou à un autre patient. En revanche, le stockage et la préparation des lignées de CSEh à la UKSCB nécessitent plusieurs tables de correspondance, allant de la mise en route du don d'embryons jusqu'à la délivrance des lignées cellulaires aux receveurs. Ainsi, la traçabilité des lignées de cellules souches embryonnaires humaines passe par un système d'identifications qui nécessite un codage successif. Les différentes étapes bien balisées intègrent des informations spécifiques illustrées par plusieurs tables de correspondances: 1) Le don d'embryons à un laboratoire est labellisé par le numéro du centre HFEA et il contient des informations de nature médicale; 2) L'établissement d'une lignée dérivée est labellisé par un nom de lignée lié à l'obtention d'une licence HFEA numérotée ; 3 ) Le traitement de la lignée par le SC implique également un codage spécifique qui regroupe l'ensemble des informations relatives à la lignée; 4) Le stockage de la lignée à la UKSCB est labellisé par un 
numéro d'accès pour la clientèle à partir duquel les données relatives aux contrôles qualité sont progressivement ajoutées au cours du processus de préparation de la lignée. Ces transferts et correspondances d'informations participent à la consistance institutionnelle de la banque, à une densification des interactions entre les différents acteurs, ainsi qu'à un estompage de ce que l'on peut a priori définir comme les frontières de la UKSCB. On arrive donc à une institution dense, forte avec des frontières peu solides (Schemeil, 2009).

Cette traçabilité biologique est également compliquée par les impératifs de sécurité sanitaire, question particulièrement prégnante en ce qui concerne les préparations de lignées orientées dans une optique de clinique. Pour cette filière, des réflexions de la UKSCB sont menées en partenariat avec les organismes britanniques de régulation et les centres effectuant des dérivations, notamment le human embryonic stem cell coordinators (hESCCO). La multiplicité des tables de correspondance particulières aux lignées embryonnaires soulève des questionnements multiples concernant les applications cliniques. Ainsi, l'anonymat du donneur est susceptible d'entrer en conflit avec les nécessités liées à son évaluation médicale exhaustive. Le codage successif des données complexifie l'identification d'informations signifiantes pour les médecins dans l'évaluation du risque clinique, notamment les critères d'exclusion du donneur relatifs à son style de vie et à ses prédispositions génétiques. La question concomitante est l'attribution des responsabilités dans la prise de décision. Le personnel de la UKSCB s'interroge également sur le type de tableau de correspondance et d'anonymisation à établir pour permettre de remonter au donneur dans le cas d'informations défavorables portant sur la lignée cellulaire. Cela pose également la question des tests virologiques à effectuer sur les lignées cellulaires dans le cadre d'une orientation clinique. En dépit de ces interrogations et controverses procédurales, le personnel de la UKSCB tente de mettre en place des pratiques de suivi rigoureusement ritualisées afin de faire correspondre et d'aligner les produits biologiques à un système de codage d'informations. Cette production documentaire cantonnée à l'espace de la UKSCB est gouvernée par un système d'enregistrement régulier et bureaucratisé, anonyme, mais traçable, donnant ainsi une représentation légitime de pratiques stériles et moralement acceptables. Par sa neutralité bureaucratique, on peut dire que la banque incarne un régime de véridiction (Martin, 2008).

Il est nécessaire pour la banque de rester à l'extérieur d'éventuels conflits d'intérêts avec les personnes susceptibles de déposer ou d'utiliser les lignées. Cela est particulièrement vrai pour les compagnies qui essaient d'optimiser des procédés de culture, souvent en liens avec les chercheurs qui ont dérivé des lignées cellulaires. De larges collaborations de type International Stem Cell Initiatives sont favorisées au sein desquelles la banque figure comme un acteur parmi d'autres dans la constitution de brevets et de «testing research». Ces opérations forment un cas de figure où les compagnies s'adressent directement à la banque pour essayer de nouveaux milieux de culture ou caractériser des lignées de CSEh. On voit ici que la circulation, le stockage des échantillons biologiques et des données les concernant sont rattachés à la standar- 
disation des méthodes, à la dissémination des connaissances et à la ratification de nouveaux paramètres. Cette institutionnalisation ramifiée constitue un dispositif d'inscription sociale, un cas d'étude adapté pour tester le croisement de travaux émanant des science studies avec des approches néo-institutionnalistes (Jasanoff, 2004).

\section{LA UKSCB: UNE MISE EN MÉMOIRE SÉLECTIVE ET DURABLE DE L'INFORMATION}

La nature prospective de la banque nationale britannique et l'établissement rigoureux d'un archivage de données fixant des correspondances étendues depuis le donneur, la dérivation de lignées, jusqu'à l'équipe recevant le produit biologique donnent une valeur biologique aux produits (Mitchell et Waldby, 2010). La mise en mémoire de l'information est sélective et durable. Comme nous allons le montrer, la banque intègre certains types cellulaires et interdit l'entrée d'autres produits embryonnaires. Elle assure une traçabilité forte des échantillons biologiques ainsi que la production d'archives durables. Cela passe par différentes voies de régulation qui influent sur la circulation des CSEh, depuis la demande effectuée par les chercheurs britanniques et étrangers de déposer ces produits biologiques à la banque, jusqu'au dépôt, puis aux candidatures des équipes pour utiliser les lignées mises à disposition à la banque. Cette standardisation procédurale a pour effet de réduire la qualification d'échantillons biologiques en leur attribuant des propriétés bien définies. Elle les fait passer d'objet biologique singulier à l'attribution d'une qualification conventionnelle de bien commun (Thévenot, 2009).

\section{Le rôle des codes de pratiques dans le mouvement éprouvé et sélectif de préparation des lignées cellulaires}

Bien qu'elles n'aient généralement pas de valeurs légales, des règles de bonnes pratiques sont apposées à l'utilisation des lignées de cellules souches embryonnaires, ce qui contribue à stabiliser leur mode de gouvernance. Ainsi, le Code of Practice développé par le SC est un code volontaire de régulation des pratiques faisant l'objet de révisions régulières. Il définit le cadre éthique et les nécessités pratiques pour l'import, l'export et l'utilisation des lignées de CSEh et il pose le rôle du SC britannique concernant la surveillance éthique de la dérivation et de l'utilisation des cellules souches. Ce code établit également les mécanismes par lesquels les chercheurs britanniques et étrangers déposent les lignées à la banque et posent candidature pour utiliser les lignées à disposition. Il joue un rôle primordial en permettant une indépendance des actions de la banque vis-à-vis des groupes d'intérêt, lui octroyant ainsi une fonction «d'intermédiaire», c'est-à-dire une fonction institutionnelle bien circonscrite dans la gouvernance générale des CSEh. De plus, en accentuant les contrôles éthiques sur les produits biologiques, le code a pour visée d'accroître la confiance du public. La UKSCB dispose également de son propre Code of Practice qui s'applique au stockage des lignées de cellules souches et à leur approvisionnement pour les recherches fondamentales et cliniques. La banque applique les bonnes pratiques en termes de validation, de 
consentement à l'éthique du don, puis de stockage, de surveillance et de délivrance des lignées. Le Local Management Committee de la banque se réunit régulièrement pour développer des stratégies concernant des questionnements techniques, notamment autour des procédures de contrôle vis-à-vis contaminations biologiques.

La circulation est également contrainte au niveau du type de matériau biologique. Certaines pratiques sont exclues suivant le Code of Practice, notamment celles qui présupposent une intervention sur le matériau embryonnaire mis en circulation. Ainsi, la banque ne peut pas stocker des embryons, des gamètes, du sang de cordon ou de la moelle; elle ne peut pas non plus dériver elle-même des CSEh, activité laissée aux équipes de recherche. Il existe également une interdiction stricte pour les scientifiques de l'UKSCB d'entreprendre des recherches fondamentales en biologie de la cellule souche ou sur des pratiques présentant un intérêt commercial, telle que la fabrication de produits de cellules souches ou de matériaux biologiques concurrents à certaines équipes. La banque ne peut pas établir un tarif de vente pour les lignées de cellules souches, elle ne peut également pas fournir de cellules souches à des fins d'applications thérapeutiques immédiates ou dans le cadre d'essais cliniques.

On voit ici le rapport particulier que ces pratiques codifiées entretiennent avec la valeur économique. La valeur du matériel biologique n'est pas ici liée à sa transformation en produit fini par l'action humaine. Elle correspond à la capacité dont dispose le personnel de la banque à définir des propriétés ainsi qu'un environnement stabilisé et codifié immédiatement identifiable par différents centres. En d'autres termes, la définition d'une valeur économique des lignées cellulaires est directement tributaire d'un cadre organisationnel d'échanges bien formalisé entre le déposant et la banque. C'est la mise en place de routines organisationnelles et leur expansion qui définit la valeur économique (Nelson, 2002) donnée aux lignées cellulaires. Ainsi, des contraintes additionnelles sont définies par le déposant de la lignée à la UKSCB, c'est-àdire le(s) propriétaire(s) de la lignée ou le laboratoire ayant effectué la dérivation. Le processus de dépôt et d'accès des lignées de CSEh dans la UKSCB passe par un triple accord faisant intervenir le déposant ", le(s) destinataire(s)/bénéficiaire(s) et la $\mathrm{UKSCB}^{10}$. Il s'agit de formulaires types standardisés fournis par la banque. Si bien que les groupes ayant effectué la dérivation doivent également candidater auprès de la UKSCB lorsqu'ils veulent entreprendre des projets sur les cellules qu'ils ont euxmêmes dérivées!

Le transfert des lignées par la UKSCB fait intervenir un grand nombre de contraintes. Dans le cas des lignées dérivées en UK, le dépôt est obligatoire. Néanmoins, de nombreuses équipes internationales, c'est-à-dire des équipes ayant obtenu des

10. UKSCB Materials Deposit Agreement (MDA) établi entre le déposant et la banque au moment du dépôt des lignées. Il donne les termes/conditions relatifs aux cellules stockées et distribuées. UKSCB Materials Access Agreement (MAA) établi entre la banque et le destinataire. Il donne les termes/conditions d'utilisation des cellules. MUL Materials Use Licence (MUL) établi entre le déposant et le destinataire. Il établi les termes/conditions relatives à la responsabilité et à la propriété intellectuelle des deux parties. Il est soumis à l'approbation du Steering Committee. 
lignées hors du territoire britannique, intègrent le processus de demande de dépôt. Pour les chercheurs britanniques souhaitant importer une lignée étrangère, cela n'est pas obligatoire, seulement nécessaire pour avoir une représentativité des lignées travaillées sur leur territoire national. Cela implique donc un accord entre l'établissement étranger ayant effectué la dérivation (terme technique pour décrire l'obtention de la lignée) et la banque avant que l'équipe britannique puisse à son tour se porter candidat à la UKSCB pour obtenir la lignée. Quoiqu'il paraisse contraignant au premier regard, cet alignement international est consenti par la majorité des équipes. Différentes motivations expliquent cette convergence.

Tout d'abord, le SC délivre un «certificat de provenance et de bonnes pratiques» aux chercheurs qui souhaitent utiliser les lignées déposées dans la UKSCB. Avant le dépôt à la banque, les lignées obtenues n'ont pas reçu la validation de ce processus d'examen éthique, qui constitue maintenant un critère de reconnaissance internationale de «bonnes pratiques» utilisable dans les publications, les demandes de financements, etc. De plus, la banque ne retient aucune propriété intellectuelle sur les lignées qu'elle stocke et distribue. Avant de délivrer la lignée à un éventuel destinataire, la banque s'assure qu'il y ait un accord entre le déposant et le destinataire pour le partage et la distribution de la propriété intellectuelle, constituée à partir des recherches menées sur cette même lignée. Le code de pratiques du SC décrit un certain nombre de principes à la base de cet accord entre les deux parties, ainsi que les contraintes éthiques, notamment l'interdiction du clonage. Les chercheurs sont également encouragés à faire intervenir la UKSCB car il est très onéreux pour chaque équipe de stocker et d'amplifier leurs propres cellules. La banque britannique prend en charge tous ces frais. La bonne qualité des lignées libérées et surtout la publicisation faite aux lignées déposées à la UKSCB forment une contrepartie au dépôt. Ainsi, la voie engagée devient celle d'un alignement à long terme des processus d'obtention des lignées sur les standards de bonnes pratiques. Comprendre le processus d'institutionnalisation de la UKSCB devient inséparable de la prise en compte des réarrangements matériels et procéduraux des objets techniques et des personnes en situation d'interaction (Pinch, 2008).

\section{Le travail de la UKSCB : archiver, tracer, amplifier, qualifier et distribuer.}

Le processus de mise en banque vise à retenir les «meilleures cellules», c'est-à-dire celles qui ont eu le moins de congélations et de traitements préalables à leur isolement in vivo. L'attention maximale est portée à la croissance des cellules constituant la premaster bank, laquelle forme un stock restreint de cellules mises en congélation. Le personnel de la banque s'assure auprès du déposant ayant fourni une lignée dérivée qu'elle se situe bien au démarrage du processus de croissance, afin de constituer une pre-master bank à partir de ce matériel biologique. À ce stade, un des échantillons est constitué en archive non utilisable. Le contrôle de la qualité est minimal; cet archivage a pour objectif d'éviter la perte de matériel biologique par les contaminations bactériennes de cellules souches ainsi que de vérifier la viabilité et la morphologie des 
cellules. Il joue également un rôle dans l'établissement d'une mémoire collective autour du dépôt de ces produits, de la production de schèmes classificatoires et du partage de données accumulées à un niveau international. Néanmoins, cette économie de l'archivage aboutissant à une pratique collective distribuée a pour effet de renforcer la division entre le centre qui compile les données (la UKSCB) et la périphérie (les équipes déposantes et candidates à l'obtention de lignées dérivées) qui exporte et reçoit les informations en retour (Bowker, 2005).

La salle de stockage dispose de trois conteneurs séparés contenant respectivement du matériel non contrôlé, du matériel en cours de contrôle et un archivage final d'ampoules stockées et cryopréservées. C'est à ce niveau que la master bank prend forme pour ensuite faire l'objet d'une caractérisation plus exhaustive en vue d'assurer la distribution ultérieure des lignées cellulaires.

L'affectation «recherche» ou «clinique» du matériel biologique intervient à ce stade de la mise en banque. Elle dépend de la manière dont la lignée cellulaire a été obtenue (type de milieux de culture, conditions GMP, etc.) et du niveau de sécurité de l'environnement initial de la lignée, notamment le risque d'une présence virale dans le matériel biologique. Là encore, le personnel de la banque [travaille] avec des organismes prestataires externes disposant d'un large spectre de tests pour détecter la présence de virus animaux en faible quantité. Cette organisation assure également la conduite de contrôle indépendamment de l'équipe ayant initialement dérivé la lignée, préservant la banque d'éventuels conflits d'intérêts.

La transition de la pre-master à la master bank est définie par deux paramètres clefs. Tout d'abord, la quantité de matériel biologique doit être suffisante et, le processus de croissance doit être amorcé. Les ampoules contenant les lignées circulent ensuite dans un espace "corridor» séparé de la première aire de stockage, où sont entreposés les milieux de culture. Les échantillons vont successivement passer dans trois petits laboratoires séparés les uns des autres pour être cultivés, amplifiés et préservés au froid. Sitôt la cryopréservation effective, ils retournent dans la première aire de stockage et y sont maintenus. Ces échantillons forment la base de la première banque de distribution.

Le matériel de la pre-master et de la première banque de distribution est cultivé suivant les consignes du déposant. La deuxième banque de distribution est marquée par des changements dans le processus de préparation ou au niveau de la méthodologie initiale. À ce point de la mise en banque, des expérimentations sont réalisées. Tout changement dans la croissance des lignées est communiqué au déposant. La garantie de mise en mémoire des lignées cellulaires est contrôlée à différentes étapes de la banque. Il est vérifié que le profil de la banque de distribution soit similaire à celui de la banque pré-master et master. S'il y a des changements observés, la banque ne détruit pas le matériel, elle spécifie simplement les modifications de critères relevés à chaque étape de la mise en banque et distribue le matériel en signalant ce changement. Des échantillons provenant des deux banques de distribution sont également archivés. L'originalité organisationnelle de la UKSCB relève de la définition des usages «à venir » 
des cellules souches définis au moment de leur dépôt par le producteur de la lignée. Si la banque constitue des stocks de cellules pour éventuellement les utiliser dans le cadre d'essais cliniques, la réserve est considérée par la banque comme une matière brute, une substance de départ. La banque ne travaille pas suivant l'objectif de faire un produit adapté à des conditions cliniques particulières. C'est au producteur que revient le travail de croissance et de manufacture du matériel en un produit final, utilisable et éventuellement adaptable à un usage de soins. La banque refuse actuellement de travailler sur des produits différenciés de CSEh et elle ne s'inscrit pas dans une logique de produits finis. En retour, toutes publications où interviennent des lignées stockées à la UKSCB impliquent que la banque soit remerciée.

En plus des contrôles destinés aux banques de lignées cellulaires de grade «recherche», les banques conçues pour des lignées cellulaires de grade «clinique» s'intègrent dans un programme exhaustif de contrôle de la qualité avec des contrôles virologiques obligatoires et des études de caractérisation des lignées. Les contrôles effectués ne visent pas simplement à vérifier les lignées réceptionnées, mais ils fournissent également des informations complémentaires sur la nature des lignées ellesmêmes. Le personnel de la banque ancre des relations durables avec les stem cell therapy product units car ils fournissent des outils de contrôles additionnels relatifs à la validation de protocoles, à l'installation de compagnies démarrant des thérapies et à l'évaluation de produits. Ces organismes assurent ainsi la continuation du processus de contrôle et de l'assurance de la qualité jusqu'au produit final.

Tout au long du processus de stockage, les lignées cellulaires doivent conserver les caractéristiques des lignées déposées, notamment la morphologie, le panel de marqueurs des cellules souches (ISCI), le caryotype (c'est-à-dire le profil génétique) et le contrôle d'identité. Elles doivent conserver plus particulièrement la capacité de se différencier en différents types cellulaires tout en restant stables en culture. Les contrôles à apposer ne font pas consensus entre les équipes de chercheurs britanniques et la banque. Certains veulent tester toutes les cellules souches, d'autres une seule partie de l'échantillon. Néanmoins, tous les acteurs britanniques reconnaissent la nécessité de bâtir des standards communs. Ce travail de réception, d'archivage, de traçabilité, d'amplification et de distribution partagée est un exemple de mouvement institutionnel conduisant à l'adhésion autour d'un projet d'étalonnage et de gouvernance des CSEh, lui-même en constitution...

\section{DU CONCEPT DE TRANSFERT INSTITUTIONNEL À LA DÉFINITION D'UNE CATÉGORIE DE MOUVEMENT}

En braquant le projecteur sur la circulation et la production de données liées aux CESh, nous avons voulu montrer le rôle central de la UKSCB dans la gouvernance internationale concernant ces produits. Nous l'avons appréhendé, non pas en tant qu'organisation artificiellement délimitée par des frontières sur lesquelles tout le monde s'accorderait, mais plutôt comme une catégorie de mouvement d'individus, de produits et d'informations (Tournay, 2009b). La traçabilité des procédures, 
l'archivage des échantillons et l'apposition d'un contrôle qualité standardisé sont des conditions nécessaires au paramétrage des lignées hétérogènes de CSEh et à la constitution d'une gouvernance étendue autour de ces produits. Cette stabilisation procédurale conduit paradoxalement à rendre invisible, les standards responsables de la consolidation de cette même gouvernance (Star et Lampland, 2009). La UKSCB constitue un bon exemple d'agencement institutionnel progressivement internationalisé par une économie particulière des flux de circulation des échantillons biologiques et des traces numériques associées à ce mouvement. Cet angle d'attaque, qui consiste à appréhender le processus de différenciation institutionnelle comme une catégorie particulière de mouvement d'individus, de produits biologiques et de traces numériques, nous amène ici à emboîter le pas à la contribution de Dumoulin et Saurugger (2011), consacrée à aux perspectives théoriques appliquées aux processus de transfert.

À partir des enseignements de ce cas d'étude, nous proposons de travailler le concept de transfert institutionnel comme une catégorie particulière de mouvement que l'on pourrait définir ainsi: «collecter, centraliser, contraindre, étalonner et répéter». Cette catégorie particulière de mouvement favorise le processus de concernement (Callon, Lascoumes et Barthe, 2001) à partir duquel les personnes et les équipes déposantes sont de plus en plus nombreuses à participer aux négociations de leur matériel biologique et à le faire représenter par la UKSCB. Si les équipes britanniques sont légalement obligées d'envoyer leurs lignées dérivées à la UKSCB, ce mouvement de collecte se globalise, en dépit des procédures administratives contraignantes, par le ralliement volontaire et abondant d'équipes étrangères. On voit ici que l'environnement s'adapte à la UKSCB autant que la banque s'adapte à son environnement (March et Olsen, 1998). Dès lors, il devient difficile d'argumenter en termes de dynamiques institutionnelles internes ou de pressions exogènes puisqu'on observe davantage une écologie de pratiques sans véritables frontières; celles-ci apparaissent et sont progressivement normalisées par la mise en œuvre d'une traçabilité et d'un formatage singulier de l'opération de collecte des échantillons. C'est pourquoi, dans ce tissu social dénué de coutures (Tournay, 2009b), plutôt que de partir de l'ancrage territorial de la UKSCB ou des rapports d'autorité inclus dans ses frontières, la notion de mouvement paraît plus adaptée pour décrire la consistance institutionnelle de la UKSCB ainsi que son internationalisation progressive. Cette institutionnalisation doit avant tout être entendue comme un système dynamique de coopérations multilatérales dépourvu d'intentionnalités dans les résultats recherchés. Ce système, en quelque sorte, «horizontalise» les relations entre les acteurs (Saez, 1997), en sortant du cadre classique de la valeur économique pour fonder une économie de l'échange non marchand. Le multilatéralisme intervient ici comme un format irréductible de la coopération internationale à partir duquel peuvent être conceptualisés d'autres agencements complexes tels que des organisations internationales (Petiteville, 2009). Aussi, l'institutionnalisation incarne un système adaptatif complexe (Olsen, 2009) dont l'équilibre se voit renégocié en permanence par l'intrusion de nouveaux produits à réguler. 


\section{REMERCIEMENTS}

Cette étude a été financée par l'Agence de la biomédecine ainsi que par l'Agence nationale de la recherche (THERACELS - édition 2008) et les Instituts de recherche en santé du Canada.

\section{RÉSUMÉ}

L'objectif de cette contribution est d'étudier le lien entre l'organisation matérielle confinée de recherches émergentes autour du vivant, leur consolidation disciplinaire et le déploiement d'espérances collectives à grande échelle. Le cas d'étude est la United Kingdom Stem Cell Bank, décrite comme une architecture performative dotée d'une efficacité organisationnelle et jouant un rôle clé dans la gouvernance internationale de la manipulation des cellules souches embryonnaires humaines. L'enjeu consiste à montrer que la structuration de cet espace confiné et stérile, nécessaire au maintien des cellules souches embryonnaires humaines, est également celle d'un espace de coopérations multilatérales et internationalisées autour de ces produits biologiques. Pour étudier cette tension scalaire, la différenciation institutionnelle de la UK Stem Cell Bank est appréhendée comme une catégorie de mouvement d'individus, de produits biologiques et de données les concernant. En intégrant les perspectives récemment développées autour des policy transfer studies, cette contribution met l'accent sur le mouvement régulier, répété et centralisé de collecte de cellules d'origine humaine vers cet espace confiné, ainsi que sur les procédures d'étalonnage et d'enregistrement des données pour expliquer le succès de ce modèle local à grande échelle.

Mots clés: institutions, cellules souches, standardisation, transfert institutionnel, approche pragmatique

\section{ABSTRACT}

The objective of this paper is to examine the relationship between the confined material organization of emerging biological researches, its consolidation into a discipline and the arising of collective hopes on a broad scale. The case under study is the United Kingdom Stem Cell Bank, described as a successfully performing and effectively organized "architecture" that plays a key role in the international regulation of the manipulation of human embryonic stem cells. The argument is to show that the structuring of this confined and sterile space necessary to the maintenance of human embryonic stem cells has also been the creation of a space of multilateral and international cooperation with regard to these biological products. In order to study the tension between these two very different scales, the institutional differentiation of the UK Stem Cell Bank is seen as a movement category of individuals, biological products and data concerning them. Integrating recently developed perspectives within the field of policy transfer studies, this article focuses on the regular, repeated and centralized movement of collecting cells of human origin toward this confined space and on the data standardization and recording procedures used in order to explain the success of this local model on a broad scale.

Key words: institutions, stem cells, standardization, institutional transfer, pragmatic approach 


\section{RESUMEN}

El objetivo de esta contribución es estudiar la relación entre la organización material confinada de las investigaciones emergentes alrededor de lo vivo, su consolidación disciplinaria y el despliegue de esperanzas colectivas a gran escala. El estudio de caso es el United Kingdom Stem Cell Bank, descrito como una arquitectura activa dotada de una eficacia organizacional y que juega un papel clave en la gobernabilidad internacional de la manipulación de las células madres embrionarias humanas. El desafío consiste en mostrar que la estructuración de este espacio confinado y estéril, necesario para el mantenimiento de las células madres embrionarias humanas, ha sido igualmente el espacio de la cooperación multilateral e internacionalizada alrededor de estos productos biológicos. Para estudiar esta tensión escalar, la diferenciación institucional del UK Stem Cell Bank es entendida como una categoría de movimiento de individuos, de productos biológicos y de datos que le conciernen. Al integrar las perspectivas recientemente desarrolladas alrededor de los policy transfer studies (estudios de políticas de transferencia), esta conclusión hace énfasis en el movimiento regular, repetido y centralizado de recolección de células de origen humano hacia este espacio confinado, así como en los procedimientos de calibración y registro de los datos para explicar el éxito de este modelo local a gran escala.

Palabras clave: instituciones, células madres, estandarización, transferencia institucional, enfoque pragmático

\section{BIBLIOGRAPHIE}

Bowker, G. (2005), Memory Practices in the Sciences, Londres, MIT Press.

Brown, N. (2003), «Hope Against Hype - Accountability in Biopasts, Presents and Futures», Science Studies, vol. 16, $\mathrm{n}^{\circ} 2$, p. 3-21.

Callon, M. , P. Lascoumes et Y. Barthe (2001), Agir dans un monde incertain. Essai sur la démocratie technique, Paris, Seuil.

Dumoulin, L. et S. SAurugger (2010), «Faisons le point sur les policy transfer studies, Analyse critique et perspectives", Critiques internationales, vol. 2, $\mathrm{n}^{\circ}$ 48, p. 9-24.

Eriksson, L., N. Stephens, A. Webster (2008), «Stem Cell Spaces, Places and Flows», New Genetics and Society, vol. 27, no 2, p. 83-85.

Franklin, S. (2005), «Stem Cells R US: Emergent Life Forms and the Global Biological», Global Assemblages - Technology, Politics, and Ethics as Anthropological Problems, USA, Blackwell Publishing, p. 59-78.

Gaudilliere, J.-P. et H.-J. Rheinberger (2004), The Mapping Cultures of Twentieth Century Genetics, Londres, Routledge.

Hastings, M. (1999), «La constellation imaginaire du service public», S. Decreton (dir.), Service public et lien social, L'Harmattan, Paris.

Hauriou, M. (1892), Précis de droit administratif et de droit public, Paris, Dalloz-Sirey.

Hilgartner, S. et S. Brandt-Rauf (1994), «Data Access, Ownership, and Control. Toward Empirical Studies of Access Practices», Science Communication, 15, nº 4, p. 355-372.

Hoeyer, K. (2010), «An Anthropological Analysis of European Union (EU) Health Governance as Biopolitics: The Case of the EU Tissues and Cells Directive», Social Science \& Medicine, 70, p. 18671873.

Healy, L., C. Hunt, L. Young et G. Stacey (2005), «The UK Stem Cell Bank: Its Role as a Public Research Resource Centre Providing Access to Well-Characterized Seed Stocks of Human Stem Cell Lines ", Advanced Drug Delivery Reviews, vol. 57, n 13, p. 1981-1988. 
Hilgartner, S. (1995), «Biomolecular Databases: New Communication Regimes for Biology?», Science Communication, 17, p. 240-263.

Hunt, C. (2007), «The Banking and Cryopreservation of Human Embryonic Stem Cells», Transfusion Medicine and Hemotherapy, 34, p. 293-304.

Jasanoff, S. (dir.) (2004), "Ordering Knowledge, Ordering Society», States of Knowledge - The CoProduction of Science and Social Order, Londres et New York, Routledge.

Lascoumes, P. et Le Gales, P. (2005), Gouverner par les instruments, Paris, Presses de Sciences Po.

March, J. et J.- P. Olsen (1998), «The Institutional Dynamics of International Political Orders ", International Organization, vol. 52, n 4, p. 943-969.

Martin, P., N. Brown, A. Turner (2008), "Capitalizing Hope: the Commercial Development of Umbilical Cord Blood Stem Cell Banking», New Genetics and Society, vol. 27, n² 2, p. 127-143.

Mitchell, R. et C. Waldby (2010), «National Biobanks: Clinical Labor, Risk Production, and the Creation of Biovalue», Science, Technology, \& Human Values, 35 (3), p. 330-355.

Nelson, R. (2002), «Bringing Institutions Into Evolutionary Growth Theory», Journal of Evolutionary Economics, vol. 17, p. 17-28.

Olsen, J. (2003), «Towards a European Administrative Space?», Journal of European Public Policy, vol. 10, no 4 , p. $506-531$.

Olsen, J. (2009), «Change and Continuity: an Institutional Approach to Institutions of Democratic Government», European Political Science Review, vol. 1, n 1, p. 3-32.

Peerbaye, A. (2004), La construction de l'espace génomique en France: La place des dispositifs expérimentaux, thèse de doctorat de l'École normale supérieure de Cachan.

Petiteville, F. (2009), Le multilatéralisme, Paris, LGDJ Montchrestien.

Pinch, T. (2008), «Technology and Institutions: Living in a Material World», Theor Soc, 37, p. 461- 483.

Rabinow, P. (1994), "Artificiality and Enlightenment: From Sociobiology to Biosociality», Essays on the Anthropology of Reason, Princeton, Princeton University Press, p. 91-112.

Rheinberger, H.-J. (1997), Toward a History of Epistemic Things: Synthesizing Proteins in the Test Tube, Standford, Standford University Press.

Rio, K.M. et O.H. Smedal (2008), «Totalization and Detotalization. Alternatives to Hierarchy and Individualism», Anthropological Theory, vol. 8, n³, p. 233-254.

SAEZ, G. (1997), «Les politiques publiques comme système de coopération. Une lecture de l'approche conventionnaliste à travers H. S. BECKer (et quelques autres)», J.-P. GAUdin et G. Novarina (dir.), Politiques publiques et négociation - multipolarités, flexibilités, hiérarchies, Paris, Éditions du CNRS, p. 19-43.

Saurugger, S. (2009), Théories et concepts de l'intégration européenne, Paris, Presses de Sciences Po.

Schemeil, Y. (2009), «Estomper les frontières, instituer le monde: L'OMC, un coopérateur marginal?», Les frontières dans tous leurs états. Les relations internationales au défi de la mondialisation, $\mathrm{P} . \mathrm{DE}$ Senarclens (dir.), Bruxelles, Emile Bruylant.

Stacey, G. (2004), «Validation of Cell Culture Media Components», Human Fertility, vol. 7, n 2, p. 113118.

Star, S.- L. et M. LAmpland (dir.) (2009), «Reckoning with Standards», Standards and their Stories How Quantifying, Classifying, and Formalizing Practices Shape Everyday Life, Ithaca \& Londres, Cornell University Press, p. 34.

Stephens, N., Atkinson, P., Glasner, P. (2008), «The UK Stem Cell Bank as Performative Architecture», New Genetics and Society, vol. 27, n 2, p. 87-98.

The International Stem Cell Banking Initiative (2009), «Consensus Guidance for Banking and Supply of Human Embryonic Stem Cell Lines for Research Purposes », Stem Cell Rev and Rep., vol. 5, p. 301-314.

Thevenot, L. (2009), «Governing Life by Standards: A View from Engagements », Social Studies of Science, vol. $39, \mathrm{n}^{\circ} 5$, p. $793-813$.

Tournay, V. (2007), La Gouvernance des innovations médicales, Paris, PUF. 
Tournay, V. (2008), «From Standards to Concerted Programs of Collective Action: The Standardization Process of Cell Therapy in France. Pragmatist Contribution to the Sociology of Innovation ", Social Theory and Health, 6, p. 220-238.

Tournay, V. (dir.) (2009 a), «Ce que revendiquer veut dire. Éléments pour une pragmatique de la monstration ", Militantisme médical et fabrique des politiques de santé, numéro spécial Quaderni, 68, p. 5-12.

Tournay, V. (2009 b), Vie et mort des arrangements sociaux. De l'origine des institutions, Paris, PUF.

Tournay, V. et A. Leibing (dir.) (2010), «Les technologies de l'espoir: Un cadre théorique pour des raisons pratiques», Les technologies de l'espoir: la fabrique d'une histoire à accomplir, Laval, PUL.

Webster, A., L. Eriksson (2008), «Governance-by-Standards in the Field of Stem Cells: Managing Uncertainty in the World of "Basic Innovation" ", New Genetics and Society, vol. 27, n 2, p. 99-111.

White, J. (2010), «European Integration by Daylight», Comparative European Politics, 8, p. 55-73. 